\title{
Correction of Camptocormia Using a Cruciform Anterior Spinal Hyperextension Brace and Back Extensor Strengthening Exercise in a Patient With Parkinson Disease
}

\author{
Byung Kook Ye, MD ${ }^{1}$, Hyoung-Seop Kim, $\mathrm{MD}^{2}$, Yong Wook Kim, MD \\ ${ }^{1}$ Department of Rehabilitation Medicine and Research Institute of Rehabilitation Medicine, \\ Yonsei University College of Medicine, Seoul; ${ }^{2}$ Department of Physical Medicine and Rehabilitation, \\ National Health Insurance Service Ilsan Hospital, Goyang, Korea
}

\begin{abstract}
Parkinson disease, one of the most common neurodegenerative diseases, is characterized by cardinal motor features including bradykinesia, rigidity, resting tremor, postural instability, freezing gait, and fatigue. Of these, postural instability in the form of hyperflexion of the thoracolumbar spine upon standing and walking that disappears on recumbent positioning is called camptocormia. Many different trials have been conducted on the treatment of camptocormia, including physiotherapy, corsets, medications, and deep brain stimulation. However, there is insufficient evidence as to which treatment modality is the most valid in terms of effectiveness, cost, safety, and patient satisfaction. In this study, we present a patient whose symptom of camptocormia was effectively resolved using a cruciform anterior spinal hyperextension (CASH) brace and back extensor strengthening exercise which was modified through follow-ups based on a short-term outpatient setting for proper application with minimal discomfort. The patient was satisfied with the amount of correction provided by the brace and exercise.
\end{abstract}

Keywords Camptocormia, Parkinson disease, Posture

\section{INTRODUCTION}

Camptocormia, a term first used in 1999, is defined as

Received April 15, 2014; Accepted July 8, 2014

Corresponding author: Hyoung-Seop Kim

Department of Physical Medicine and Rehabilitation, National Health Insurance Service Ilsan Hospital, 100 Ilsan-ro, Ilsandong-gu, Goyang 410-719, Korea

Tel: +82-31-900-0137, Fax: +82-31-900-0343, E-mail: rekhs@nhimc.or.kr

(c) This is an open-access article distributed under the terms of the Creative Commons Attribution Non-Commercial License (http://creativecommons. org/licenses/by-nc/3.0) which permits unrestricted noncommercial use, distribution, and reproduction in any medium, provided the original work is properly cited.

Copyright $\odot 2015$ by Korean Academy of Rehabilitation Medicine an abnormal extreme forward flexion of the thoracolumbar spine of $\geq 45^{\circ}$ that is often manifested in patients with Parkinson disease (Fig. 1A, B) [1]. Camptocormia is suspected to be caused by axial dystonia of the spinal muscles or focal myopathy $[2,3]$. This involuntary postural abnormality is a disabling phenomenon during the course of Parkinson disease with comorbidities, such as back pain, gait disturbance, and postural instability. Sakas et al. [4] presented two cases of restoring erect posture in patients with idiopathic camptocormia using electrical stimulation of globus pallidus internus (GPi), suggesting that camptocormia may result from abnormal oscillation highly confined to the GPi cluster. In addition, many 

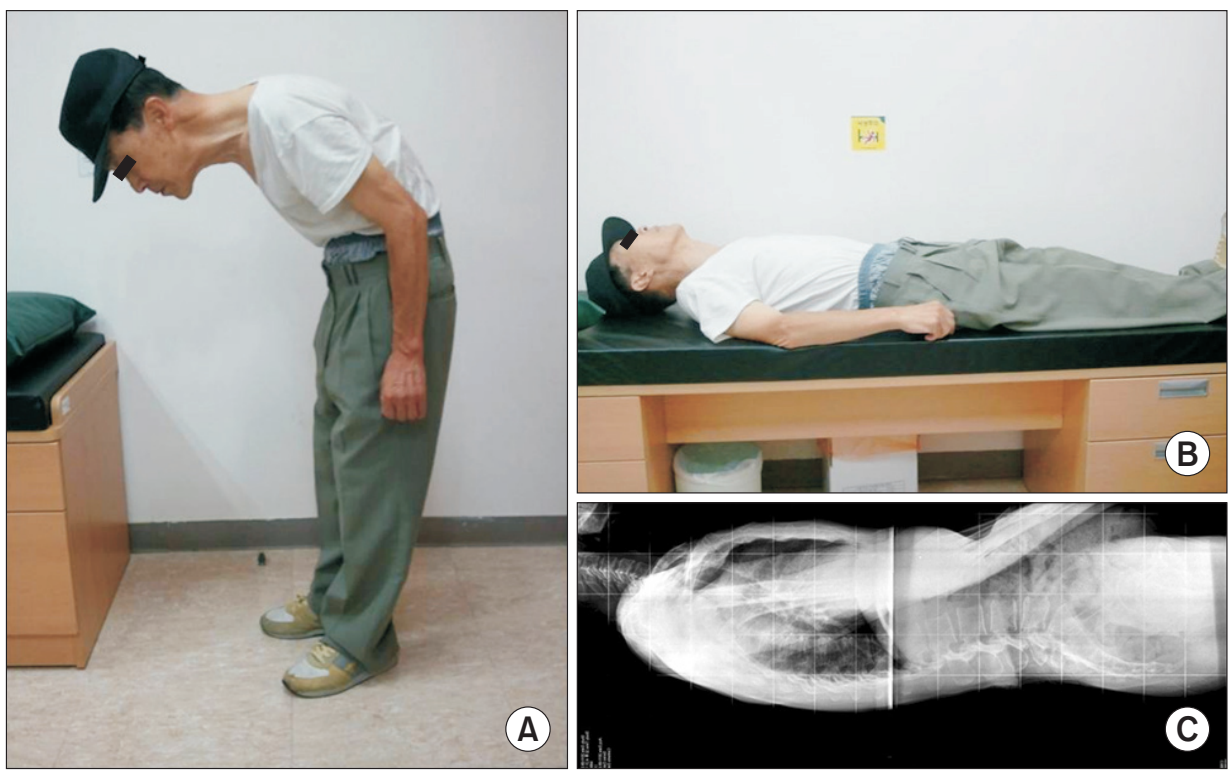

Fig. 1. (A) Hyperflexion of the thoracolumbar spine observed on the initial outpatient visit. (B) Hyperflexion of the thoracolumbar spine resolved by recumbent positioning. (C) No evidence of compression fracture seen on plain X-ray (whole spine lateral view, supine position).

types of treatments have been implemented, including pharmacological therapy as well as physiotherapy and corsets. However, no effective treatment has been widely acknowledged yet [5]. Azher and Jankovic [6] reported a good response of levodopa in the motor symptoms of 11 patients with Parkinsonism, but with no effect on their camptocormia. Meanwhile, there are several studies with promising results of orthosis effect in patients with osteoporotic kyphosis. It was reported that 31 patients with spinal osteoporosis who wore a weighted kypho-orthosis for six months showed a $11 \%$ decrease in their angle of kyphosis [7]. In two studies published by Sinaki et al. [8] in 2005, groups of patients with spinal osteoporosis wearing a weighted kypho-orthosis with exercise showed an improvement in their kyphotic posture, body balance, gait velocity, and cadence. However, the pathogenesis of camptocormia in spinal osteoporosis is quite different from that of Parkinsonism. Studies described above are conducted on patients with osteoporotic camptocormia.

Here, we present a case of camptocormia that was effectively corrected in a patient diagnosed with Parkinson disease using a cruciform anterior spinal hyperextension (CASH) brace and back extensor strengthening exercises.

\section{CASE REPORT}

The patient was a 70-year-old man who initially visited the Department of Neurology at a tertiary training hospital on May 9, 2009 for weakness accompanied by tremor in both legs that began in early 2009. He was diagnosed with Parkinson disease with regular follow-up at an outpatient setting for medication management. Symptoms of involuntary truncal flexion first began in June 2011 without response to medication dosage control. He was referred to the Department of Physical Medicine and Rehabilitation of our hospital on October 12, 2011. As there was sustained lumbar flexion during anti-gravity activities but none on recumbent positioning, camptocormia, an involuntary abnormal forward flexion of thoracolumbar spine and anterocollis, was suspected (Fig. 1).

The ambulatory ability of the patient was measured by 'Functional Ambulation Category' (FAC) with category 3 and by 'time up and go test' at 16 seconds. The patient refused muscle enzyme level, MRI, and electromyography studies for economic reasons. Moreover, the patient showed no symptomatic response to the following medication: Requip (ropinirole $8 \mathrm{mg}$, once a day, daily, for March 3, 2011-November 16, 2011) and Madopar (levodopa $200 \mathrm{mg}$ and benserazide $50 \mathrm{mg}$, three times a day, daily, for March 7, 2011-present). Application of a backpack loaded with two standard bricks at $3.8 \mathrm{~kg}$ (center of gravity was to located on the level of inferior angle of the scapulae and length of the string was adjusted for total contact in order to induce the extension of the trunk similar to a weighted kypho-orthosis) was intolerable due to heavy weight (Fig. 2). Therefore, a CASH brace was applied on October 19, 2011 in order to provide a resistive limitation to the forward flexion of the thoracolumbar 


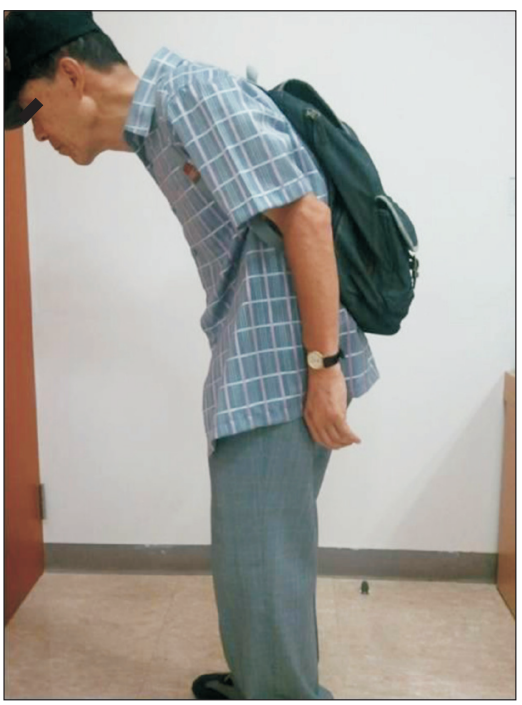

Fig. 2. Extension of the thoracolumbar spine partially achieved with application of a heavily loaded backpack, but the weight was intolerable to the patient.

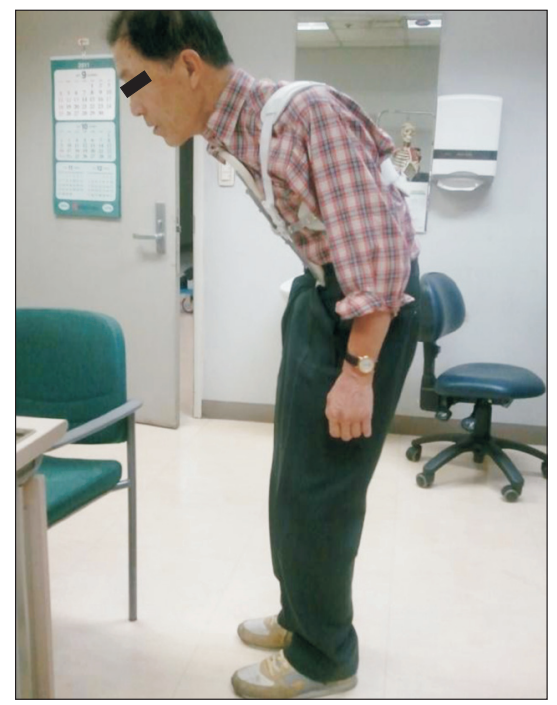

Fig. 3. Camptocormia was still observed after the initial application of the cruciform anterior spinal hyperextension brace.
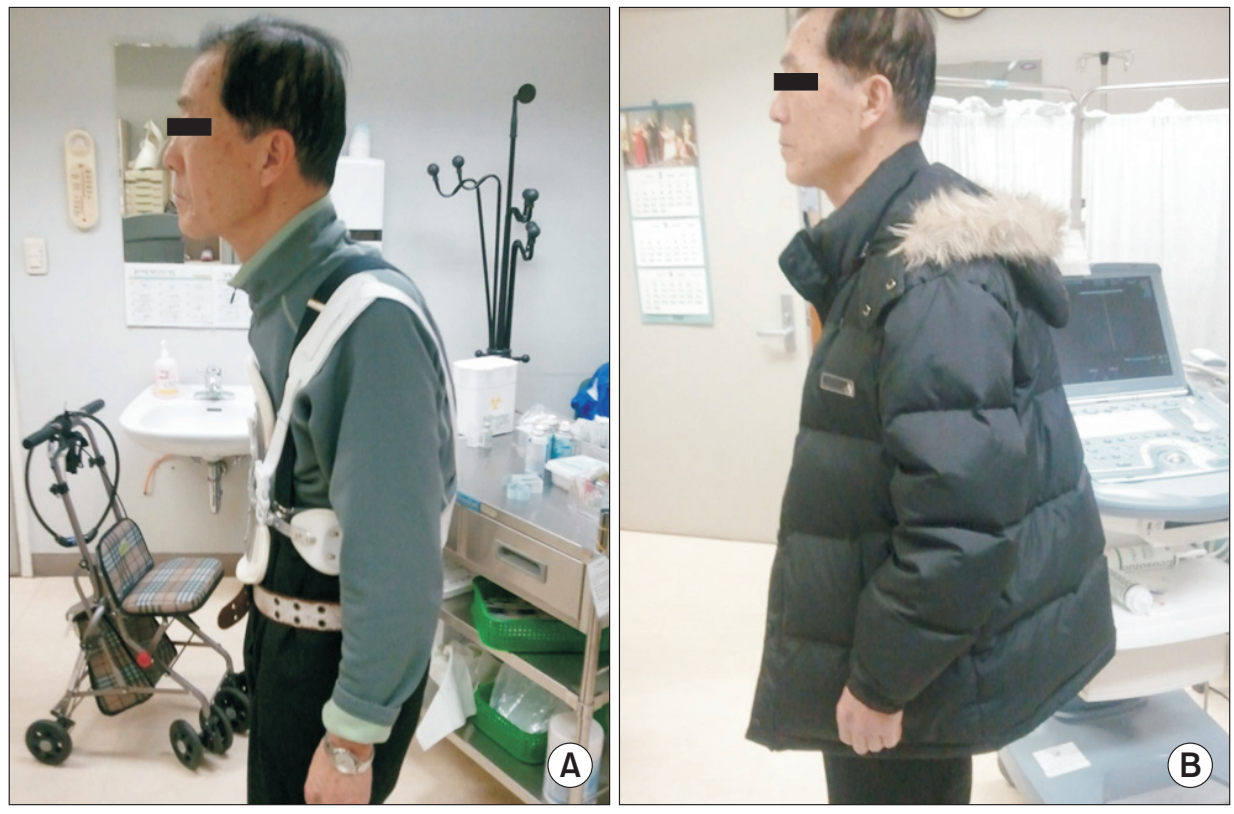

Fig. 4. (A, B) Correction of camptocormia after a serial of outpatient-based follow-ups and modifications. spine (Fig. 3). The patient was instructed to perform daily back and neck extensor strengthening exercises for 30 minutes. Through many short-term outpatient visits, the CASH brace was modified to fit the patient without discomfort from any improper pressure (Fig. 4). After four months, the patient was able to walk independently with the support of CASH brace through holding an involuntary flexion of the thoracolumbar spine. His ambulatory ability was also improved. The FAC changed from category 3 to category 5 . The time up and go test improved from 16 seconds to 9 seconds. The patient has been followed up for 5 months. He remains satisfied with the effect of the brace for correcting the uncomfortable postural deformity. In the end, he was able to maintain a good posture without the brace. 


\section{DISCUSSION}

The pathogenesis of camptocormia is not yet wellunderstood. The underlying pathophysiology of camptocormia is suggested to be of either central or peripheral origins, axial dystonia, or focal myopathy of the spinal muscles $[1,3]$. Forward bending of the spine results from a weakness of the paravertebral muscles or over-activity of the abdominal muscles. However, no evidence is available to support either factor in the development of camptocormia.

As surgical approaches consisting of deep brain stimulation (DBS) of GPi have corrected the truncal and cervical flexion, the central nervous system involvement, specifically GPi, is the most probable central anatomical lesion for camptocormia. Although interfastigial cerebellum and bilateral midbrain tegmentum play important roles in the maintenance of erect posture and bipedalism, restoration of erect posture has only been achieved by DBS of the GPi, further suggesting its involvement in camptocormia [4]. However, despite the rapid and dramatic effects of DBS in correcting camptocormia, it is limited due to its major surgical procedure with risks that might accompany as any other major neurosurgeries. In addition, of 15 cases that have been successfully treated with DBS, the average age of patient at onset was 40.57 years, and that of surgery was 49.07 years. Therefore, most patients are relatively young. Of these patients, primary patients with a greater number of motor symptoms, including dystonia, showed better results. There was only one case of successful treatment among patients above the age of 70 years, which is an age group with relative contraindication of DBS [9]. Antiparkinson medications including levodopa, dopamine agonists, amantadine and anticholinergics are infrequently mildly beneficial and mostly ineffective $[5,10]$. Antispastic medications also have no obvious benefit. A botulinum toxin injection is mildly effective in four out of nine patients for up to three months [6]. It has been suggested that camptocormia may be caused by myopathic changes [3] due to evidences, such as myopathic electromyographic findings and myopathic histologic changes. Similar to camptocormia, anterocollis, the dropped head in patients with Parkinsonism, is also closely related with myopathic changes of neck extensor muscles [2]. The number of patients mentioned in the two studies above was 11 with average age of 71.45 years, which is relatively older than those in the DBS group. Because of those differences, age is assumed to be a key factor in pathophysiology. Furthermore, the relative contraindication of DBS involves the age group above 70 years, and the absolute contraindication of DBS is associated with dementia which explains the cause of the difference.

Although physiotherapy accompanied by a corset and brace is feasible, it is often unsatisfactory to patients because corset and braces are uncomfortable due to inappropriate prescription, fitting, or donning. For that reason, we have followed our patient in short-term courses on an outpatient basis and made modifications based on his feedback. In our case, the patient complained of pain around the sternum. To avoid sternal area pain and discomfort caused by rubbing between the brace and sternum, it is helpful to perform back extensor strengthening exercises. Although the patient complained of discomfort in the pubic area in this period, the symptom disappeared immediately as the trunk flexion was improved. Our patient is currently able to wear the brace comfortably throughout the day without complaints of excess pressure on any specific site that may lead to pain or discomfort with effective correction of camptocormia during standing and ambulation. Although the effects may be less dramatic than those achieved by DBS, it seems valid to apply a brace before undergoing such procedures considering its safety and cost.

In conclusion, despite previous reports on the mediocre effects of applying brace to correct camptocormia in patients with Parkinsonism, we suggest that a $\mathrm{CASH}$ brace with back extensor strengthening exercise can be an effective and satisfactory method of treatment if serial short-term follow-ups with frequent modifications to the brace are provided. However, it may be a limitation that we report only one case. Therefore, case series or a large trial should be considered in further studies.

\section{CONFLICT OF INTEREST}

No potential conflict of interest relevant to this article was reported.

\section{REFERENCES}

1. Doherty KM, van de Warrenburg BP, Peralta MC, Si- 
lveira-Moriyama L, Azulay JP, Gershanik OS, et al. Postural deformities in Parkinson's disease. Lancet Neurol 2011;10:538-49.

2. Askmark H, Eeg-Olofsson K, Johansson A, Nilsson P, Olsson Y, Aquilonius S. Parkinsonism and neck extensor myopathy: a new syndrome or coincidental findings? Arch Neurol 2001;58:232-7.

3. Schabitz WR, Glatz K, Schuhan C, Sommer C, Berger C, Schwaninger M, et al. Severe forward flexion of the trunk in Parkinson's disease: focal myopathy of the paraspinal muscles mimicking camptocormia. Mov Disord 2003;18:408-14.

4. Sakas DE, Panourias IG, Stavrinou LC, Boviatsis EJ, Themistocleous M, Stathis P, et al. Restoration of erect posture in idiopathic camptocormia by electrical stimulation of the globus pallidus internus. J Neurosurg 2010;113:1246-50.

5. Melamed E, Djaldetti R. Camptocormia in Parkinson's disease. J Neurol 2006;253 Suppl 7:VII14-16.

6. Azher SN, Jankovic J. Camptocormia: pathogenesis, classification, and response to therapy. Neurology 2005;65:355-9.

7. Pfeifer M, Begerow B, Minne HW. Effects of a new spinal orthosis on posture, trunk strength, and quality of life in women with postmenopausal osteoporosis: a randomized trial. Am J Phys Med Rehabil 2004;83:17786.

8. Sinaki M, Brey RH, Hughes CA, Larson DR, Kaufman KR. Significant reduction in risk of falls and back pain in osteoporotic-kyphotic women through a Spinal Proprioceptive Extension Exercise Dynamic (SPEED) program. Mayo Clin Proc 2005;80:849-55.

9. Reese R, Knudsen K, Falk D, Mehdorn HM, Deuschl G, Volkmann J. Motor outcome of dystonic camptocormia treated with pallidal neurostimulation. Parkinsonism Relat Disord 2014;20:176-9.

10. Bloch F, Houeto JL, Tezenas du Montcel S, Bonneville F, Etchepare F, Welter ML, et al. Parkinson's disease with camptocormia. J Neurol Neurosurg Psychiatry 2006;77:1223-8. 